\title{
Age differences in veridical and false recall are not inevitable: The role of frontal lobe function
}

\author{
KARIN M. BUTLER \\ University of New Mexico, Albuquerque, New Mexico \\ MARK A. MCDANIEL \\ Washington University, St. Louis, Missouri \\ COURTNEY C. DORNBURG \\ University of New Mexico, Albuquerque, New Mexico \\ AMANDA L. PRICE \\ Elizabethtown College, Elizabethtown, Pennsylvania \\ and \\ HENRY L. ROEDIGER III \\ Washington University, St. Louis, Missouri
}

\begin{abstract}
The relationship of neuropsychological measures of frontal lobe function to age differences in false recall was assessed using the Deese/Roediger-McDermott associative false memory paradigm (Deese, 1959; Roediger \& McDermott, 1995). As other studies have found, older adults were less likely to correctly recall studied items and more likely to falsely recall highly related but nonpresented items than were younger adults. When older adults were divided based on a composite measure of frontal lobe functioning, this age difference was found only for low frontal lobe functioning individuals. High frontal lobe functioning older adults and young adults had equivalent levels of false recall, as well as equivalent levels of veridical recall. These results suggest that age differences in memory may be due to declines in frontal lobe function. More important, our findings indicate that declines in veridical recall and increases in false recall are not an inevitable consequence of aging.
\end{abstract}

Memory for events is not always veridical. Sometimes a characteristic of an episode is recalled even though it was experienced in a different context, or sometimes even when the information was never experienced at all. Age-related increases in memory distortions and illusions have been reported across a wide set of experimental paradigms (e.g., Cohen \& Faulkner, 1989; Dywan \& Jacoby, 1990; Koutstaal \& Schacter, 1997; Multhaup, De Leonardis, \& Johnson, 1999; Rankin \& Kausler, 1979), including the Deese/Roediger-McDermott (DRM) paradigm (Balota et al., 1999; Intons-Peterson, Rocchi, West, McLellan, \& Hackney, 1999; Norman \& Schacter, 1997; Tun, Wingfield, Rosen, \& Blanchard, 1998).

In the DRM paradigm (Deese, 1959; Roediger \& McDermott, 1995), participants are presented with lists of

This research was supported by National Institute of Aging Grant AG17481 to M.A.M. and H.L.R. The findings were presented in 2002 at the 49th Annual Meeting of the Psychonomic Society in Kansas City, MO. We thank the volunteers from the New Mexico Aging Process Study (funded by National Institute on Aging Grant AG02049) for their participation. We also thank Elizabeth Glisky and Patrick Davidson for providing the equation used to calculate frontal lobe function scores. Correspondence concerning this article should be addressed to K. M. Butler, Department of Psychology, Logan Hall, University of New Mexico, Albuquerque, NM 87131 (e-mail: kmbutler@unm.edu). words (e.g., bed, rest, awake, . . .) that share a strong association with a critical theme word (e.g., sleep) that is not presented on the list (Roediger, Watson, McDermott, \& Gallo, 2001). Participants often incorrectly remember the highly associated, but not presented, critical item, typically with high levels of confidence (e.g., Roediger \& McDermott, 1995).

A potential indicator of the underlying cause of the age difference in false memory is that older adults report less difference between the qualitative characteristics of studied and critical items than do young adults. These results suggest that older adults fail to encode or to retrieve itemspecific details for studied items (Norman \& Schacter, 1997; see also Intons-Peterson et al., 1999; Koutstaal \& Schacter, 1997; Tun et al., 1998), and this idea is consistent with the literature reporting age-related deficits in source monitoring (e.g., Hashtroudi, Johnson, \& Chrosniak, 1990).

Furthermore, frontal lobe (FL) dysfunction has been implicated as the neurological source of monitoring and attentional control difficulties experienced by older adults (Balota, Dolan, \& Duchek, 2000; Raz, 2000). FL functioning is negatively correlated with older adults' reliance (appropriately or inappropriately) on knowledge of previous choices and stereotype information in making source attributions (Mather \& Johnson, 2000; Mather, Johnson, 
\& De Leonardis, 1999), a type of false recognition. Likewise, an FL patient has been shown to have high false alarm rates to related (but not to unrelated) distractors on a memory test (Curran, Schacter, Norman, \& Galluccio, 1997), and patients with damage to the frontal lobes demonstrate confabulation, the creation of false memories of experiences that never really happened to them (Moscovitch, 1989).

The considerations above converge on the hypothesis that FL functioning may be critically related to age-related increases in false recall. An initial report failed to find correlations between false recall and performance on digit span, word fluency, and mental control tasks, tests thought to primarily measure frontal functioning (Balota et al., 1999). However, these tasks may reflect processing in other cortical areas as well, and the reliability of these tasks used individually may not be high enough.

Accordingly, we adopted a more extensive way of assessing FL functioning in older adults. On the basis of Glisky, Polster, and Routhieaux's (1995) factor-analytic work with neuropsychological tests, we assessed frontal function with five tests that have been individually related to FL function. These measures were not related to a second distinct factor composed of measures of item memory; they related instead to medial temporal lobe function. The strength of using a composite measure of FL functioning is that the score reflects the shared variance of the entire set of FL tests. Variance in the composite FL function measure is more likely to reflect variance in cognitive processes specifically associated with the frontal lobes than would variance associated with any individual test score. (See Glisky et al., 1995, for details.)

In this study, we used the method of Glisky et al. (1995) to categorize older adults as high or low FL functioning to determine whether FL functioning may contribute to age differences in false memory in the DRM paradigm. If the frontal lobes are responsible for controlling attention and/or source monitoring in such a way that false memories can be distinguished from true memories, then we thought it possible that older adults with low FL function scores would show greater false recall of the nonpresented theme words than both their high-functioning counterparts and younger adults. Such a pattern would represent an important boundary for age-related differences in false memory. The idea tested here is that the increased susceptibility to memory illusions in older adults is carried by those with relatively low frontal function.

\section{METHOD}

\section{Participants}

Eighteen young and 36 older adults participated in this experiment. As can be seen in Table 1, young adults had fewer years of education $[t(52)=5.64]$ and lower scores on the Shipley Vocabulary Test (Shipley, 1940) $[t(50)=7.62]$ than did older adults, indicating that the older adult group was generally high functioning.

Neuropsychological assessments. The five measures used to calculate the composite FL function scores were the number of categories achieved on the modified Wisconsin Card Sorting Test
Table 1

Participant Characteristics

\begin{tabular}{|c|c|c|c|c|c|c|c|c|}
\hline & \multicolumn{2}{|c|}{$\begin{array}{l}\text { Younger } \\
(n=18)\end{array}$} & \multicolumn{2}{|c|}{$\begin{array}{c}\text { Older } \\
(n=36)\end{array}$} & \multicolumn{2}{|c|}{$\begin{array}{l}\text { Low FL } \\
\text { Function } \\
(n=17)\end{array}$} & \multicolumn{2}{|c|}{$\begin{array}{l}\text { High FL } \\
\text { Function } \\
(n=19)\end{array}$} \\
\hline & $M$ & $S D$ & $M$ & $S D$ & $M$ & $S D$ & $M$ & $S D$ \\
\hline Age & 19.0 & 0.8 & 76.4 & 5.1 & 77.5 & 3.3 & 75.5 & 6.3 \\
\hline Educ & 13.7 & 1.2 & 16.5 & 2.5 & 16.5 & 2.9 & 16.5 & 2.2 \\
\hline Vocab & 26.3 & 4.3 & 35.3 & 3.8 & 33.8 & 3.3 & 36.7 & 3.7 \\
\hline MMSE & & & 28.5 & 1.2 & 28.0 & 1.3 & 29.0 & 1.0 \\
\hline GDS & & & 3.1 & 2.2 & 3.8 & 2.3 & 2.5 & 2.0 \\
\hline FL function scores & & & & & -0.80 & 0.40 & 0.36 & 0.27 \\
\hline
\end{tabular}

Note-FL, frontal lobe; Educ, years of education; Vocab, Shipley Vocabulary Test; MMSE, Mini-Mental State Exam; GDS, Geriatric Depression Scale. Vocabulary scores were available for only 16 of the 18 young subjects.

(modified WCST; Hart, Kwentus, Wade, \& Taylor, 1988); the total number of words generated for the letters $F, A$, and $S$ on the Controlled Oral Word Association Test (COWAT; Spreen \& Benton, 1977); the Arithmetic score from the Wechsler Adult Intelligence ScaleRevised (WAIS-R; Wechsler, 1981); and the Mental Control and Backward Digit Span scores from the Wechsler Memory Scale-III (Wechsler, 1997). For each participant, a composite measure of FL function was calculated as the average of age-adjusted $z$ scores on each of the frontal measures. The FL function scores were calculated using an equation that was derived in the same way as has been reported previously (Glisky et al., 1995; Glisky, Rubin, \& Davidson, 2001; McDaniel, Glisky, Rubin, Guynn, \& Routhieaux, 1999). The equation that we used was based on a new normative sample tested in E. Glisky's lab (personal communication, October, 2001). Among the older adult participants, 17 with FL function scores above zero were placed in the high-FL-function group $(M=0.36$, range $=$ 0.04 to 1.20 ) and 19 scoring below zero were placed in the low-FLfunction group $(M=-0.80$, range $=-1.43$ to -0.03$) .{ }^{1}$ Four participants were missing scores on one of the assessments, so in those cases FL function scores were calculated using the four available scores. In addition, 29 older adults had completed the standard version of the WCST (Heaton, Chelune, Talley, Kay, \& Curtiss, 1993) a year before this study. Rather than administer the task again under the easier instructions (Hart et al., 1988), we used the scores from the standard version. To ensure that the increased difficulty of the standard version did not change the classification of the older adults, FL-function scores were calculated with and without the WCST. Three individuals whose FL function classification changed from high to low when the standard WCST was included in the calculation were excluded from the sample. ${ }^{2}$

As can be seen in Table 1, the low- and high-FL-function groups both had a mean of 16.5 years of education. Low-FL-function individuals had lower vocabulary scores than did the high-FL-function group $[t(34)=2.48]$, but their scores were still higher than those of young adults $[t(31)=5.58]$. All the older adults were screened for depression and dementia. Anyone scoring 10 or above on the Geriatric Depression Scale was excluded for possible depression (Brink et al., 1982). There was a trend toward the low-FL-function group having higher mean depression scores than their higher functioning counterparts $[t(34)=1.73, p=.09]$. Anyone scoring 25 or below on the Mini-Mental State Exam was excluded for possible dementia (MMSE; Folstein, Folstein, \& McHugh, 1975). The lowFL-function group had lower scores on the MMSE than did the high-FL-function group $[t(34)=2.63]$.

\section{Materials}

Thirty-six DRM lists of 15 words each were chosen to represent a range of probabilities of false recall of the critical item. Twenty- 
three lists chosen from the Stadler, Roediger, and McDermott (1999) norms had high probabilities of false recall ranging from .34 to .65 . Thirteen lists chosen from Roediger et al. (2001) had low probabilities of false recall ranging from .01 to .10 . This list difference did not enter into any interactions with either age group and item type or FL function group and item type. Therefore, we collapsed the data across this variable.

\section{Procedure}

Participants were tested in two 2-h sessions that were scheduled 2 weeks apart. In Session 1, in a task unrelated to present purposes, participants first spent an hour performing and imagining simple actions. Then, following the completion of the vocabulary test and a break, participants studied and were tested on the first set of 12 DRM lists. During Session 2, participants spent 30 min completing a memory test for the actions that they had performed during Session 1 . Then they were presented with the remaining two sets of DRM lists (each set comprised 12 lists). Following the DRM lists in Session 2, older, but not younger, adults were asked to complete four additional measures - the Geriatric Depression Scale, the Arithmetic subscale from the WAIS-R, the MMSE, and the COWAT - if they had not completed them recently.

In each session, the participants were instructed that they would study a list of words and that after each list, they would be given 2 min to recall the words from the list in any order. They were told that they should only write down words that they were reasonably sure had been presented on the list and that they should not guess. Before beginning the first set of experimental lists, the participants practiced the procedure with a list of 15 U.S. states.

Then the experimental-list testing began. The 15 words in each list were presented visually, one at a time for $2 \mathrm{sec}$ each, in order from strongest to weakest associative strength. Each study list was followed by a recall test for $2 \mathrm{~min}$. A recognition test followed each set of 12 lists. Recognition test performance did not vary significantly by either age or FL function group. Although the direction of group differences in means was the same as for the recall data, to conserve space we do not report the recognition data.

\section{RESULTS}

For all analyses, the rejection level for inferring statistical significance was set at .05 . Because of time limitations, experimenter error, and fatigue, complete recall data from all three sets of DRM lists were not available for 5 young, 1 low-FL-function, and 2 high-FL-function individuals. When the analyses reported below were performed only with individuals having complete data, the patterns of means and significant effects were identical. In addition, performance across the three sets of DRM lists was analyzed. List set did not interact with age group or FL function group, indicating that the patterns of data reported below were the same in each set of lists. Therefore, we report the results from analyses including all participants collapsed across all sets of lists.

Consistent with previous studies, we found that younger adults were more likely to correctly recall the studied items (.62) than were older adults (.53) $[t(52)=$ $3.25]$. In addition, younger adults were less likely to intrude the critical items (.20) than were older adults (.29), with this difference being marginally significant $[t(52)=$ $1.85, p=.07]$.

Of critical interest was whether the older adults' performance varied according to FL functioning. Recall data are presented in Figure 1. We first compared correct and

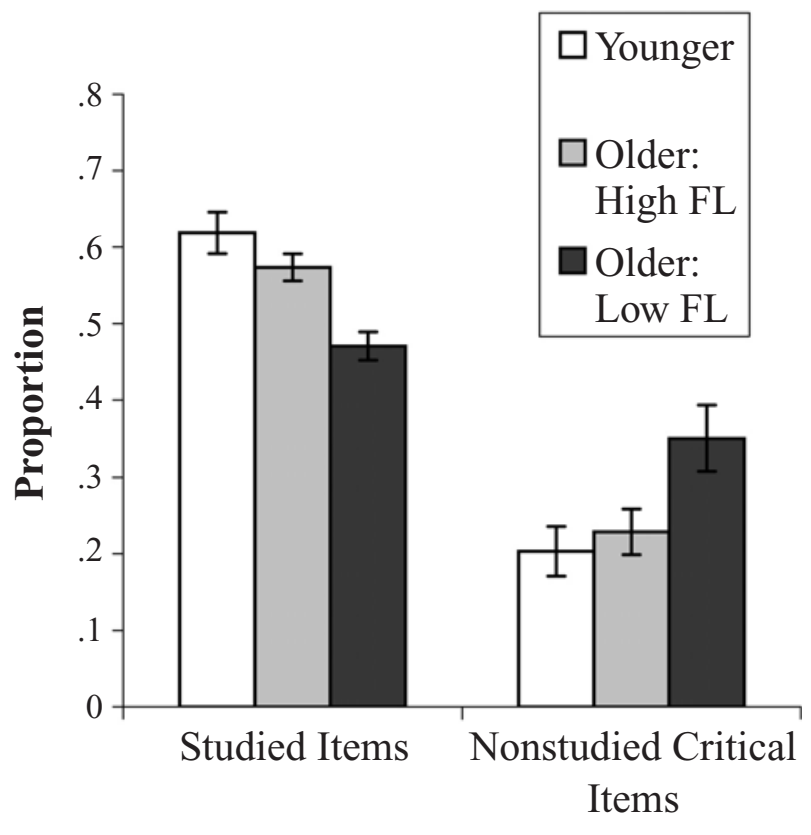

Figure 1. Probability of veridical and false recall as a function of subject group.

false recall by FL function group for older adults. The low-FL-function group was less likely to recall studied words (.47) than was the high-FL-function group (.57) $[t(34)=4.04]$ and was also more likely to intrude critical items (.35) than was the high-FL-function group (.23) $[t(34)=2.38]$. These results were reflected in a significant correlation between FL function scores and correct recall (.52) and in a trend toward a negative correlation between FL function scores and false recall $(-.29, p=.08)$.

Remarkably, the performance of the high-FL-function older adults was no different from that of the younger adults. Analyses confirmed this impression: There were no significant differences between the younger and highFL-function older adults when we compared veridical recall (.62 and .57 , respectively) $[t(35)=1.41]$ and false recall (.20 and .23 , respectively) $[t(35)=0.58]$. Age differences in veridical $[t(33)=4.47]$ and false $[t(33)=$ 2.76 ] recall were found only when younger and low-FLfunction older adults were compared.

Intrusions of noncritical items during recall were also examined. Note that these data are mean numbers of intrusions per list, not proportions. Although the pattern for incorrect recall of noncritical items was similar to that found for the critical items, the mean number of intrusions per list was not significantly different for younger $(0.21)$ and older $(0.32)$ adults $[t(52)=1.28]$ nor for high- $(0.23)$ and low- (0.42) FL-function older adults $[t(34)=1.66]$.

\section{DISCUSSION}

Older adults were more likely to incorrectly recall critical items than were younger adults even though they had 
lower levels or veridical recall, replicating research by others (Balota et al., 1999; Intons-Peterson et al., 1999; Norman \& Schacter, 1997; Tun et al., 1998). The present investigation reveals that this pattern holds only for older adults who perform poorly on a battery of tests that have been related to FL function. The performance of high-FL-function older adults in our study was indistinguishable from the performance of younger adults. Not only did the high-FL-function older adults incorrectly recall critical items at the same level as the younger adults did, they also had levels of correct recall that were comparable to those of younger adults.

Although researchers have speculated that FL functioning may be related to age-related exaggeration of false memory, this is the first report establishing a significant relationship between performance on neuropsychological tests of FL functioning and false recall. Previous attempts to correlate FL function and false memory may have been hindered by the low reliability of individual measures of FL functioning (e.g., Balota et al., 1999). Similarly, only one of our five frontal measures correlated significantly with false recall (Arithmetic, $r=$ -.40 ); the remaining four did not (WCST, $r=-.14$; COWAT, $r=-.10$; Backward Digit Span, $r=-.18$; Mental Control, $r=-.30$ ). Using several neuropsychological tests to derive a composite measure of FL functioning produced a measure of FL functioning that was more sensitive to variations in false recall. The present support for frontal involvement in false memory is indirect, however, because it rests on the assumption that the FL function score is reflective of the functioning of the frontal lobes. Still, in a result converging on this assumption and the present recall findings, Curran, Schacter, Johnson, and Spinks (2001) found that late, frontal memory-related event-related potential activity in young adults was associated with better discrimination of the critical and studied items on a recognition test.

Our results parallel a finding in another false memory paradigm that used a cued recall procedure with categorized lists. Using the same composite measure of FL functioning to compare high- and low-FL-function older adults with younger adults, Meade and Roediger (2002) found an age difference in the false recollection of critical items for low-, but not for high-FL-function older adults. Taken in concert with the present findings, these results provide a foundation for the view that older adults are more likely to produce false memories because of declines in FL functioning (Balota et al., 1999; Norman \& Schacter, 1997).

Contrary to the reports of Glisky and colleagues (Glisky et al., 1995; Glisky et al., 2001), we found that veridical recall was associated with FL function scores. Possibly our sample of high-FL-function older adults may have also scored well on tests of item memory associated with medial temporal lobe (MTL) functioning. To inform this possibility, we computed MTL function scores (Glisky et al., 1995) on the basis of standardized memory test scores available for 9 low- and 13 high-FL-function older adults from our sample. These subgroups still differed on measures of veridical and false recall (average correct recall of .44 and .58 and false recall of .35 and .23, respectively, for low- and high-FL-function groups). However, the difference between the average MTL scores of the low- $(-0.508)$ and high- $(-0.055)$ FL-function groups was not significant $[t(20)=-1.36]$. In addition, both FL function groups had below-average MTL scores, suggesting that differences between the groups in MTL function are not the cause of differences in veridical recall. A more likely explanation for the relationship between FL function scores and veridical recall is that strategic processes in the frontal lobes aid in remembering lists of associated words (cf. Shimamura, 1995), whereas Glisky's studies used forced choice recognition of sentences as the item-memory task.

Our research also suggests that within a relatively high-frontal-function subgroup of older adults, age differences in both veridical and false recall are not substantial, at least in the immediate recall of lists of related words. Thus, declines in recall for older adults may be less widespread than is commonly accepted (Craik \& Jennings, 1992). It must be borne in mind that although there were no behavioral differences between the younger and the high-FL-function older adults in this study, the lack of behavioral differences does not necessarily translate into the same neurological functioning. Several neuroimaging studies have shown that older adults who were equated with young adults on behavioral measures, such as working memory and source memory performance, nonetheless recruited different areas of the brain during task performance (Cabeza, Anderson, Locantore, \& McIntosh, 2002; for a brief review, see Reuter-Lorenz, 2002). These findings suggest that what distinguishes highfrom low-performing older adults is not necessarily the absence of age-related decline, but rather the ability of the high-performing group to compensate for age-related changes in neurological functioning (Cabeza et al., 2002; Reuter-Lorenz, 2002).

Although poorer veridical recall and increased false recall are consistent findings in the aging literature, we identified a group of older adults who performed like younger adults in both veridical and false recall. These individuals scored well on a group of tests associated with FL function that measure working memory and executive control processes. Older adults that score well on this battery are also better at source monitoring (Glisky et al., 1995; Glisky et al., 2001; Henkel, Johnson, \& De Leonardis, 1998) and less reliant on stereotype information (Mather et al., 1999), suggesting that declines in frontally mediated cognitive processes, such as source monitoring and strategic processing, play a prominent role in age-related deficits in false memory.

\section{REFERENCES}

Balota, D. A., Cortese, M. J., Duchek, J. M., Adams, D., Roediger, H. L., III, McDermott, K. B., \& Yerys, B. E. (1999). Veridical and false memories in healthy older adults and in dementia of the Alzheimer's type. Cognitive Neuropsychology, 16, 361-384.

Balota, D. A., Dolan, P. O., \& DucheK, J. M. (2000). Memory changes 
in healthy older adults. In E. Tulving \& F. I. M. Craik (Eds.), The Oxford handbook of memory (pp. 395-409). Oxford: Oxford University Press.

Brink, T. L., Yesavage, J. A., Lum, O., Heersema, P., Adey, M. B., \& Rose, T. L. (1982). Screening tests for geriatric depression. Clinical Gerontologist, 1, 37-44.

Cabeza, R., Anderson, N. D., Locantore, J. K., \& McIntosh, A. R. (2002). Aging gracefully: Compensatory brain activity in highperforming older adults. NeuroImage, 17, 1394-1402.

Cohen, G., \& FAulKNer, D. (1989). Age differences in source forgetting: Effects on reality monitoring and on eyewitness testimony. Psychology \& Aging, 4, 10-17.

CRAIK, F. I. M., \& JENNINGS, J. M. (1992). Human memory. In F. I. M. Craik \& T. A. Salthouse (Eds.), The handbook of aging and cognition (pp. 51-110). Hillsdale, NJ: Erlbaum.

Curran, T., Schacter, D. L., Johnson, M. K., \& Spinks, R. (2001). Brain potentials reflect behavioral differences in true and false recognition. Journal of Cognitive Neuroscience, 13, 201-216.

Curran, T., Schacter, D. L., Norman, K. A., \& Galluccio, L. (1997). False recognition after a right frontal lobe infarction: Memory for general and specific information. Neuropsychologia, 35, 1035-1049.

DEESE, J. (1959). On the prediction of occurrence of particular verbal intrusions in immediate recall. Journal of Experimental Psychology, 58, 17-22.

DYWAN, J., \& JACOBY, L. L. (1990). Effects of aging on source monitoring: Differences in susceptibility to false fame. Psychology \& Aging, 5, 379-387.

Folstein, M. F., Folstein, S. E., \& McHugh, P. R. (1975). Mini-Mental State: A practical method for grading the state of patients for the clinician. Journal of Psychiatric Research, 12, 189-198.

Glisky, E. L., Polster, M. R., \& Routhieaux, B. C. (1995). Double dissociation between item and source memory. Neuropsychology, 9 , 229-235.

GLISKY, E. L., Rubin, S. R., \& DAVIDSON, P. S. R. (2001). Source memory in older adults: An encoding or retrieval problem? Journal of Experimental Psychology: Learning, Memory, \& Cognition, 27, 1131-1146.

Hart, R. P., KWentus, J. A., Wade, J. B., \& TAYlor, J. R. (1988). Modified Wisconsin Card Sorting Test in elderly normal, depressed and demented patients. Clinical Neuropsychologist, 2, 49-56.

Hashtroudi, S., Johnson, M. K., \& ChrosniaK, L. D. (1990). Aging and qualitative characteristics of memories for perceived and imagined complex events. Psychology \& Aging, 5, 119-126.

Heaton, R. K., Chelune, G. J., Talley, J. L., Kay, G. G., \& Curtiss, G. (1993). Wisconsin Card Sorting Test manual: Revised and expanded. Odessa, FL: Psychological Assessment Resources.

Henkel, L. A., Johnson, M. K., \& De Leonardis, D. M. (1998). Aging and source monitoring: Cognitive processes and neuropsychological correlates. Journal of Experimental Psychology: General, 127, 251-268.

Intons-Peterson, M. J., Rocchi, P., West, T., Mclellan, K., \& HACKNEY, A. (1999). Age, testing at preferred or nonpreferred times (testing optimality), and false memory. Journal of Experimental Psychology: Learning, Memory, \& Cognition, 25, 23-40.

KoutstaAl, W., \& SCHACTER, D. L. (1997). Gist-based false recognition of pictures in older and younger adults. Journal of Memory \& Language, 37, 555-583.

MATHER, M., \& Johnson, M. K. (2000). Choice-supportive source monitoring: Do our decisions seem better to us as we age? Psychology \& Aging, 15, 596-606.

MATHer, M., Johnson, M. K., \& De Leonardis, D. M. (1999). Stereotype reliance in source monitoring: Age differences and neuropsychological test correlates. Cognitive Neuropsychology, 16, 437-458.

McDaniel, M. A., Glisky, E. L., Rubin, S. R., Guynn, M. J., \& Routhieaux, B. C. (1999). Prospective memory: A neuropsychological study. Neuropsychology, 13, 103-110.

Meade, M. L., \& Roediger, H. L., III (2002, November). Age differences in illusory memories produced by forced recall. Paper presented at the 43rd Annual Meeting of the Psychonomic Society, Kansas City, MO.
Moscovitch, M. (1989). Confabulation and the frontal systems: Strategic versus associative retrieval in neuropsychological theories of memory. In H. L. Roediger III \& F. I. M. Craik (Eds.), Varieties of memory and consciousness: Essays in honour of Endel Tulving (pp. 133-156). Hillsdale, NJ: Erlbaum.

Multhaup, K. S., De Leonardis, D. M., \& Johnson, M. K. (1999). Source memory and eyewitness suggestibility in older adults. Journal of General Psychology, 126, 74-84.

NoRMAN, K. A., \& SCHACTER, D. L. (1997). False recognition in younger and older adults: Exploring the characteristics of illusory memories. Memory \& Cognition, 25, 838-848.

RANKIN, J. L., \& KAUSLER, D. H. (1979). Adult age differences in false recognitions. Journal of Gerontology, 34, 58-65.

RAZ, N. (2000). Aging of the brain and its impact on cognitive performance: Integration of structural and functional findings. In F. I. M. Craik \& T. A. Salthouse (Eds.), Handbook of aging and cognition (2nd ed., pp. 1-90). Mahwah, NJ: Erlbaum.

ReUter-Lorenz, P. A. (2002). New visions of the aging mind and brain. Trends in Cognitive Sciences, 6, 394-400.

Roediger, H. L., III, \& MCDERMOTT, K. B. (1995). Creating false memories: Remembering words not presented in lists. Journal of Experimental Psychology: Learning, Memory, \& Cognition, 21, 803-814.

Roediger, H. L., III, Watson, J. M., McDermott, K. B., \& Gallo, D. A. (2001). Factors that determine false recall: A multiple regression analysis. Psychonomic Bulletin \& Review, 8, 385-407.

Shimamura, A. P. (1995). Memory and frontal lobe function. In M. S. Gazzaniga (Ed.), The cognitive neurosciences (pp. 803-813). Cambridge, MA: MIT Press.

SHIPLEY, W. C. (1940). A self-administered scale for measuring intellectual impairment and deterioration. Journal of Psychology, 9, 371377.

Spreen, O., \& Benton, A. L. (1977). Neurosensory Center comprehensive examination for aphasia (rev. ed.). Victoria, BC: University of Victoria, Neuropsychology Laboratory.

Stadler, M. A., Roediger, H. L., III, \& McDermott, K. B. (1999). Norms for word lists that create false memories. Memory \& Cognition, 27, 494-500.

Tun, P. A., Wingfield, A., Rosen, M. J., \& Blanchard, L. (1998). Response latencies for false memories: Gist-based processes in normal aging. Psychology \& Aging, 13, 230-241.

WECHSLER, D. (1981). Wechsler Adult Intelligence Scale-Revised manual. New York: Psychological Corp.

WeCHSLER, D. (1997). Wechsler Memory Scale-III manual. New York: Psychological Corp.

\section{NOTES}

1. The healthy, community-dwelling older adults that we have administered these neuropsychological assessments to are more likely to be classified as low than as high FL functioning, indicating that our older adult sample is lower functioning on average than Glisky's normative sample, in which half are classified as having high FL function. To date, the 289 older adults we have tested at the University of New Mexico and Washington University using these neuropsychological assessments have a mean FL function score of -.338 and only 86 of the 289 are high FL functioning, i.e., have FL function scores greater than .03 .

2. Young adults were not administered this battery of assessments because this method has not been validated in young adults, and we did not have the resources to do so ourselves. In addition, it is unlikely that this battery of tasks would be sensitive to individual differences in FL functioning in young adults. Although COWAT, Mental Control, and Arithmetic performance can be as variable in young as in older adults, performance on the modified WCST is at ceiling for young adults (Cabeza, Anderson, Locantore, \& McIntosh, 2002).

(Manuscript received June 13, 2003; revision accepted for publication November 6, 2003.) 